\title{
Facile synthesis and excellent electrochemical performance of CoP nanowire on carbon cloth as bifunctional electrode for hydrogen evolution reaction and supercapacitor
}

\author{
Jiefang Song ${ }^{1,2}$, Jianyong Xiang ${ }^{1}$, Congpu $\mathrm{Mu}^{2 *}$, Bochong Wang ${ }^{2}$, Fusheng Wen ${ }^{1,2^{*}}$, Can $\mathrm{Su}^{2}$, \\ Cong Wang ${ }^{2}$ and Zhongyuan $\mathrm{Liu}^{2 *}$
}

\begin{abstract}
In this paper, we report CoP nanowires supported on carbon cloth (CC) (CoP/CC) as a bifunctional electrode for hydrogen evolution reaction (HER) and supercapacitor. CoP/CC possess an excellent electrocatalytic performance for HER, with a Tafel slope of $56 \mathrm{mV} / \mathrm{dec}$ and a low overpotential of $68 \mathrm{mV}$ to achieve a current density of 10 $\mathrm{mA} \mathrm{cm}{ }^{-2}$. Remarkably, the bifunctional CoP/CC used as electrode for supercapacitor exhibit a higher specific capacitance of $674 \mathrm{~F} \mathrm{~g} \mathrm{~g}^{-1}$ at a scan rate of $5 \mathrm{mV} \mathrm{s}^{-1}$ and maintains long-life cycling stability, retaining $86 \%$ of the initial capacitance after 10,000 cycles. CoP/CC will be a promising candidate as electrode for HER and supercapacitor.
\end{abstract}

Keywords: cobalt phosphide nanowire, hydrogen evolution reaction, supercapacitor

\section{INTRODUCTION}

Currently, most energy worldwide comes from burning fossil fuels to produce electrical energy. With the excessive exploitation of fossil fuel energy, fossil fuels will be exhausted in the near future. Furthermore, the combustion of fossil fuels has created environmental pollution for plants, animals and humans [1,2]. In order to solve the issues of fossil fuel depletion and increasing environmental problems, a rich, environmentally friendly, sustainable and high calorific value energy source is urgently required $[3,4]$. Clean energy sources have attracted extensive attention from many researchers in the past few years, such as nuclear power [5,6], solar energy [7], wind energy [8], tidal power [9] and hydrogen [10,11]. Hydrogen, as a clean and sustainable energy carrier, is considered one of the most ideal candidates to replace fossil fuels in the future $[3,12]$. Efficient hydrogen production is critical for developing hydrogen energy technology. There are many methods to produce hydrogen, such as coal gasification, partial oxidation, catalytic steam reforming, and water electrolysis $[3,13]$. Water electrolysis, which produces hydrogen via electrochemical splitting of water, has been known as the cleanest hydrogen production method. In this method, an advanced electrocatalyst plays the key role in hydrogen evolution reaction (HER) [10,14]. Currently, Pt-based metallic materials are the best catalysts for HER. However, the high cost and low abundance limits their application [15]. Therefore, the discovery of a non-precious electrocatalyst with long-life cycling stability, high activity and low cost for HER is critically important. Recently, transition metal sulfides $\left(\mathrm{WS}_{2}, \mathrm{MoS}_{2}, \mathrm{CoS}_{2}\right)[10,16]$, transition metal hydroxides $\left(\mathrm{Ni}(\mathrm{OH})_{2}, \mathrm{Co}(\mathrm{OH})_{2}\right)$ [17,18], and transition metal phosphides $\left(\mathrm{Ni}_{x} \mathrm{P}, \mathrm{Co}_{x} \mathrm{P}, \mathrm{Fe}_{x} \mathrm{P}, \mathrm{Fe}-\mathrm{CoP}\right)$ [19-23] have been investigated as efficient, low-cost and nonprecious catalysts for HER, displaying high performance. Consequently, transition metal phosphide materials have received significant attention as active HER catalysts $[19,24]$.

In order to use hydrogen energy, it is important to develop new energy storage devices. Supercapacitors have been widely promoted as promising candidates for new energy storage devices owing to their high-energy density, high-power density, long cycle life, quick charge and environmental friendliness [25-27]. According to the supercapacitor charge-storage mechanism, super-

\footnotetext{
${ }^{1}$ Hebei Key Laboratory of Microstructure Material Physics, Yanshan University, Qinhuangdao 066004, China

${ }^{2}$ State Key Laboratory of Metastable Materials Science and Technology, Yanshan University, Qinhuangdao 066004, China

* Corresponding authors (emails: congpumu@ysu.edu.cn (Mu C.), wenfsh03@126.com (Wen F), liuzy0319@yahoo.com (Liu Z))
} 
capacitors can be divided into electrochemical doublelayer capacitors and pseudocapacitors [10]. Transition metal phosphides, particularly cobalt phosphide and its derivatives, have been broadly studied as anode materials for Li-ion batteries, and electrocatalysts for HER, oxygen evolution reaction, and hydrodesulfurization and hydrodenitrogenation reactions. Recent reports have indicated that transition metal phosphides (cobalt, nickel, iron phosphides) display good performance for HER owing to their good electrical conductivity [12,20,21]. Electrical conductivity is also a critical parameter for supercapacitor anode materials. Therefore, transition metal phosphides can be used as supercapacitor anode material, which has not been reported to date.

The performance of supercapacitor and electrocatalysts for HER has been improved by the development of nanomaterials with various nanostructures, such as nanowires, nanoparticles, and nanosheets [21,24,28,29]. Furthermore, flexible electrodes will play an important role for electrocatalysts in flexible water splitting devices and supercapacitors in flexible electronic devices $[19,24,30]$. Herein, we developed a two-step strategy for synthesizing cobalt phosphide $(\mathrm{CoP})$ nanowires supported on carbon fiber cloth (CC) (CoP/CC) as electrocatalyst for HER and electrode material for supercapacitor. The CoP/CC can be quickly synthesized and showed excellent electrochemical performance. The CoP/CC exhibited excellent electrocatalytic performance for HER, with a Tafel slope of $56 \mathrm{mV} / \mathrm{dec}$ and a low overpotential of $68 \mathrm{mV}$ to achieve a current density of 10 $\mathrm{mA} \mathrm{cm}{ }^{-2}$. In addition, the CoP/CC used as electrode for supercapacitor exhibited a high specific capacitance of $674 \mathrm{~F} \mathrm{~g}^{-1}$ at a scan rate of $5 \mathrm{mV} \mathrm{s}^{-1}$, and maintained a long-life cycling stability, retaining $86 \%$ of the initial capacitance after 10,000 cycles.

\section{EXPERIMENTAL SECTION}

All reagents of analytical grade were directly used without further purification. Cobalt (II) nitrate hexahydrate (Co $\left.\left(\mathrm{NO}_{3}\right)_{2} \cdot 6 \mathrm{H}_{2} \mathrm{O}, 98 \%-102 \%\right)$, urea $\left(\mathrm{CO}\left(\mathrm{NH}_{2}\right)_{2}, 99 \%\right)$, and sodium hypophosphite $\left(\mathrm{NaH}_{2} \mathrm{PO}_{4}, 97\right.$ wt.\%) were purchased from Alfa Aesar. Ammonium fluoride $\left(\mathrm{NH}_{4} \mathrm{~F}, \mathrm{AR}\right.$, 99\%) was purchased from Tianjin Kaitong Chemical Reagent Co. (China). CC was purchased from Toray Industries. The deionized water used in all the experiments was purified by a laboratory pure water system (18.25 $\mathrm{M} \Omega \mathrm{cm}$ ).

Typically, CC was cleaned successively by acetone, ethanol, and deionized water for $15 \mathrm{~min}$ each. Then, CC and $\mathrm{HNO}_{3}$ were transferred to a water bath and main-

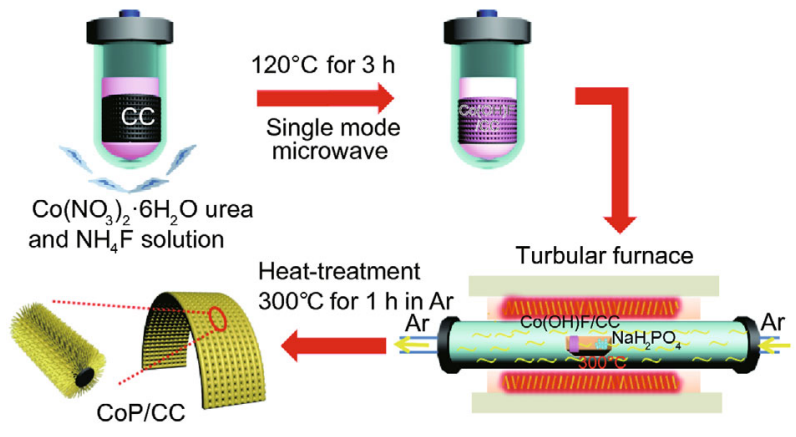

Figure 1 Schematic diagram of the synthesis process of CoP/CC.

tained at $30^{\circ} \mathrm{C}$ for $5 \mathrm{~h}$ to remove impurities on the CC surface. Finally, CC was washed repeatedly with deionized water and then dried in a drying oven at $120^{\circ} \mathrm{C}$ for $12 \mathrm{~h}$.

The synthetic process for generating $\mathrm{CoP}$ nanowires supported on CC is shown in Fig. 1. Co(OH)F/CC were first synthesized, and then $\mathrm{CoP} / \mathrm{CC}$ were fabricated by phosphorization of $\mathrm{Co}(\mathrm{OH}) \mathrm{F} / \mathrm{CC}$. Firstly, $\mathrm{Co}\left(\mathrm{NO}_{3}\right)_{2}$. $6 \mathrm{H}_{2} \mathrm{O}(0.1455 \mathrm{~g}), \mathrm{NH}_{4} \mathrm{~F}(0.0465 \mathrm{~g})$ and $\mathrm{CO}\left(\mathrm{NH}_{2}\right)_{2}(0.15 \mathrm{~g})$ were dissolved in $10 \mathrm{~mL}$ of deionized water by magnetic stirring for $20 \mathrm{~min}$. A diaphanous pink solution was obtained. A piece of CC (about $1 \times 2 \mathrm{~cm}^{2}$ in size) and the diaphanous pink solution $(10 \mathrm{~mL})$ were transferred into a specialized and sealed glass tube and heated at $120^{\circ} \mathrm{C}$ for $3 \mathrm{~h}$ with microwave irradiation in a single-mode microwave autoclave (NOVA-II, Preekem of Shanghai, China). After allowing the microwave autoclave to cool down slowly at room temperature, the $\mathrm{Co}(\mathrm{OH}) \mathrm{F} / \mathrm{CC}$ were removed and washed with deionized water and ethanol, then dried at $80^{\circ} \mathrm{C}$ for $12 \mathrm{~h}$ in a drying oven. To fabricate $\mathrm{CoP} / \mathrm{CC}, \mathrm{Co}(\mathrm{OH}) \mathrm{F} / \mathrm{CC}$ and $\mathrm{NaH}_{2} \mathrm{PO}_{4}$ were placed at either end of a crucible. Then, the crucible end with $\mathrm{NaH}_{2} \mathrm{PO}_{4}$ was placed at the upstream side of the tubular furnace. The samples were heated at $300^{\circ} \mathrm{C}$ for $1 \mathrm{~h}$ under an $\mathrm{Ar}$ atmosphere, and then naturally cooled to room temperature under the protection of an $\mathrm{Ar}$ atmosphere. Finally, CoP nanowires supported on CC ( $\mathrm{CoP} / \mathrm{CC})$ were obtained.

HER and supercapacitor measurements were performed with $\mathrm{CHI}-660 \mathrm{D}$ electrochemical workstation at room temperature, using a typical three-electrode cell. HER activity was evaluated in $0.5 \mathrm{~mol} \mathrm{~L}^{-1} \mathrm{H}_{2} \mathrm{SO}_{4}$. A graphite rod served as the reference electrode and a saturated calomel electrode (SCE) was used for the counter electrode. The potential transfer from SCE to reversible hydrogen electrode (RHE) in $0.5 \mathrm{~mol} \mathrm{~L}^{-1} \mathrm{H}_{2} \mathrm{SO}_{4}$ was 
performed by $E_{\mathrm{RHE}}=0.281+E_{\mathrm{SCE}}$. Linear sweep voltammetry (LSV) was performed at a scan rate of $5 \mathrm{mV} \mathrm{s}^{-1}$. Cyclic voltammetry (CV) measurements were carried out at $100 \mathrm{mV} \mathrm{s}^{-1}$. The supercapacitor performance of $\mathrm{CoP} /$ $\mathrm{CC}$ was measured by $\mathrm{CV}$, galvanostatic charge-discharge (GCD) measurements, and electrochemical impedance spectroscopy (EIS) in $1 \mathrm{~mol} \mathrm{~L}^{-1} \mathrm{KOH}$ solution. A platinum foil and $\mathrm{Hg} / \mathrm{HgO}$ were utilized as the reference and counter electrodes, respectively. The EIS measurements were conducted with a voltage amplitude of $5 \mathrm{mV}$, under an open-circuit potential, and in a frequency range from $100 \mathrm{kHz}$ to $0.01 \mathrm{~Hz}$. The specific capacitance $\left(C, \mathrm{~F} \mathrm{~g}^{-1}\right)$ was calculated according to $C=\int I \mathrm{~d} v / 2 m \cdot \Delta u \cdot v$, where $I$ is the current density, $v$ is the scan rate, and $m$ is the mass of active materials.

\section{RESULTS AND DISCUSSION}

The X-ray diffraction (XRD) pattern of CoP/CC and CC is shown in Fig. 2a. CC (black curve) shows two diffraction peaks, at around $25^{\circ}$ and $43^{\circ}$, which can also be observed in the XRD of CoP/CC. The diffraction peaks at $31.6^{\circ}, 35.3^{\circ}, 36.3^{\circ}, 46.2^{\circ}, 48.1^{\circ}, 52.3^{\circ}, 56.4^{\circ}$ and $56.8^{\circ}$ can be indexed to diffraction from the (011), (200), (111), (112), (211), (103), (212), and (301) planes of CoP, respectively, which is consistent with the CoP standard pattern (JCPDS No. 29-0497). The XRD results are in agreement with the literature $[14,19]$. Compared to the $\mathrm{XRD}$ of $\mathrm{Co}(\mathrm{OH}) \mathrm{F} / \mathrm{CC}$ (as shown in Fig. $\mathrm{S} 1$ ), $\mathrm{Co}(\mathrm{OH}) \mathrm{F} /$ $\mathrm{CC}$ have been completely transformed into CoP/CC via phosphorization. To further verify the complete phosphorization, the XPS spectrum of CoP/CC is provided in Fig. S2. Fig. 2b shows the scanning electron microscopy (SEM) image and corresponding elemental mapping of $\mathrm{P}$, $\mathrm{Co}$ and $\mathrm{C}$ in $\mathrm{CoP} / \mathrm{CC}$, which indicates that $\mathrm{Co}$ and $\mathrm{P}$ in

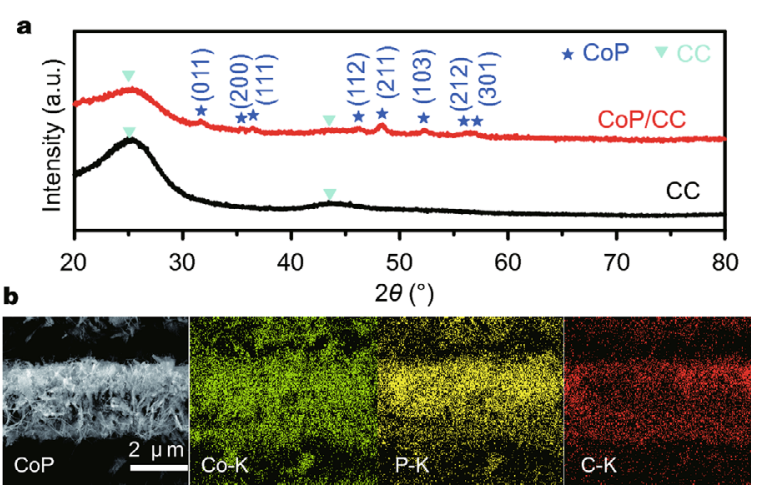

Figure 2 (a) XRD patterns of CoP/CC and CC; (b) SEM image and corresponding elemental mapping of CoP/CC.

$\mathrm{CoP} / \mathrm{CC}$ are uniformly distributed throughout the whole CoP/CC sample. The XRD and elemental mapping results verify that CoP has been uniformly grown on the CC.

The low-magnification SEM image of CoP/CC is shown in Fig. 3a. By comparison to the SEM images of CC in Fig. $\mathrm{S} 3$, Fig. 3a indicates that the $\mathrm{CoP}$ nanowires have uniformly grown on the entire CC surface. The high-magnification SEM images (Fig. 3b, c) reveal that the CoP nanowires have diameters of about $60 \mathrm{~nm}$, with lengths of a few micrometers. Fig. 3d, e show the transmission electron microscopy (TEM) images of the CoP nanowires. The CoP nanowires have a rough surface with plenty of pores, which is attributed to gas release and dehydration during $\mathrm{Co}(\mathrm{OH}) \mathrm{F} / \mathrm{CC}$ annealing. The low- and highmagnification SEM images of $\mathrm{Co}(\mathrm{OH}) \mathrm{F} / \mathrm{CC}$ are shown in Fig. S4, indicating the morphology of the $\mathrm{Co}(\mathrm{OH}) \mathrm{F}$ nanowires. It is clear that the nanowire morphology has changed from solid $\mathrm{Co}(\mathrm{OH}) \mathrm{F}$ to porous $\mathrm{CoP}$ in the phosphorization process. Further different magnification SEM images of CoP/CC have been presented in Fig. S5,

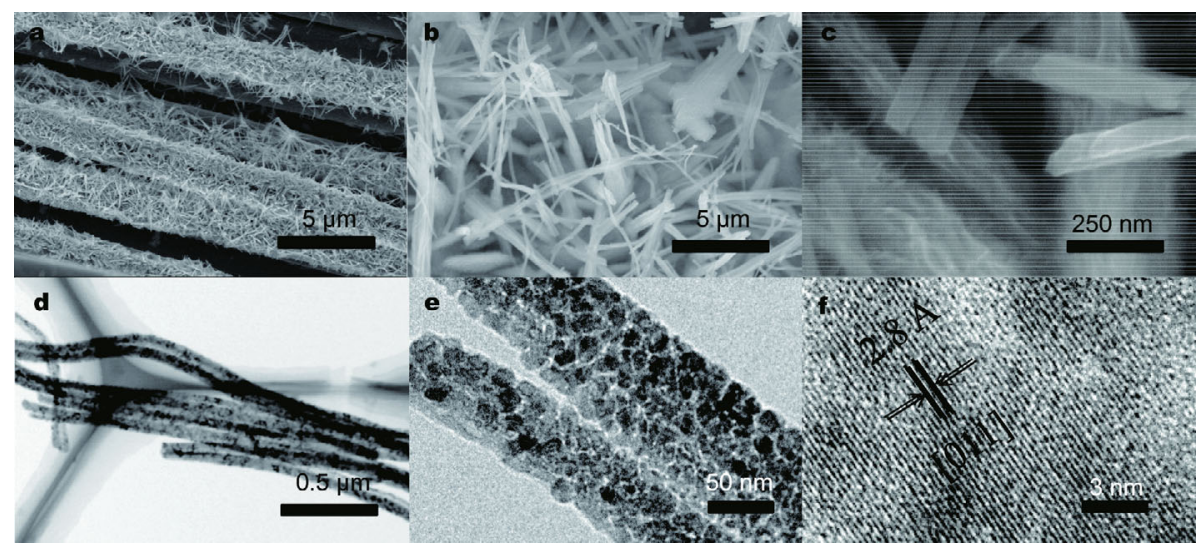

Figure 3 Characterization of CoP/CC morphology. (a-c) SEM images at different magnifications; (d, e) TEM images at different magnifications; (f) HRTEM image. 

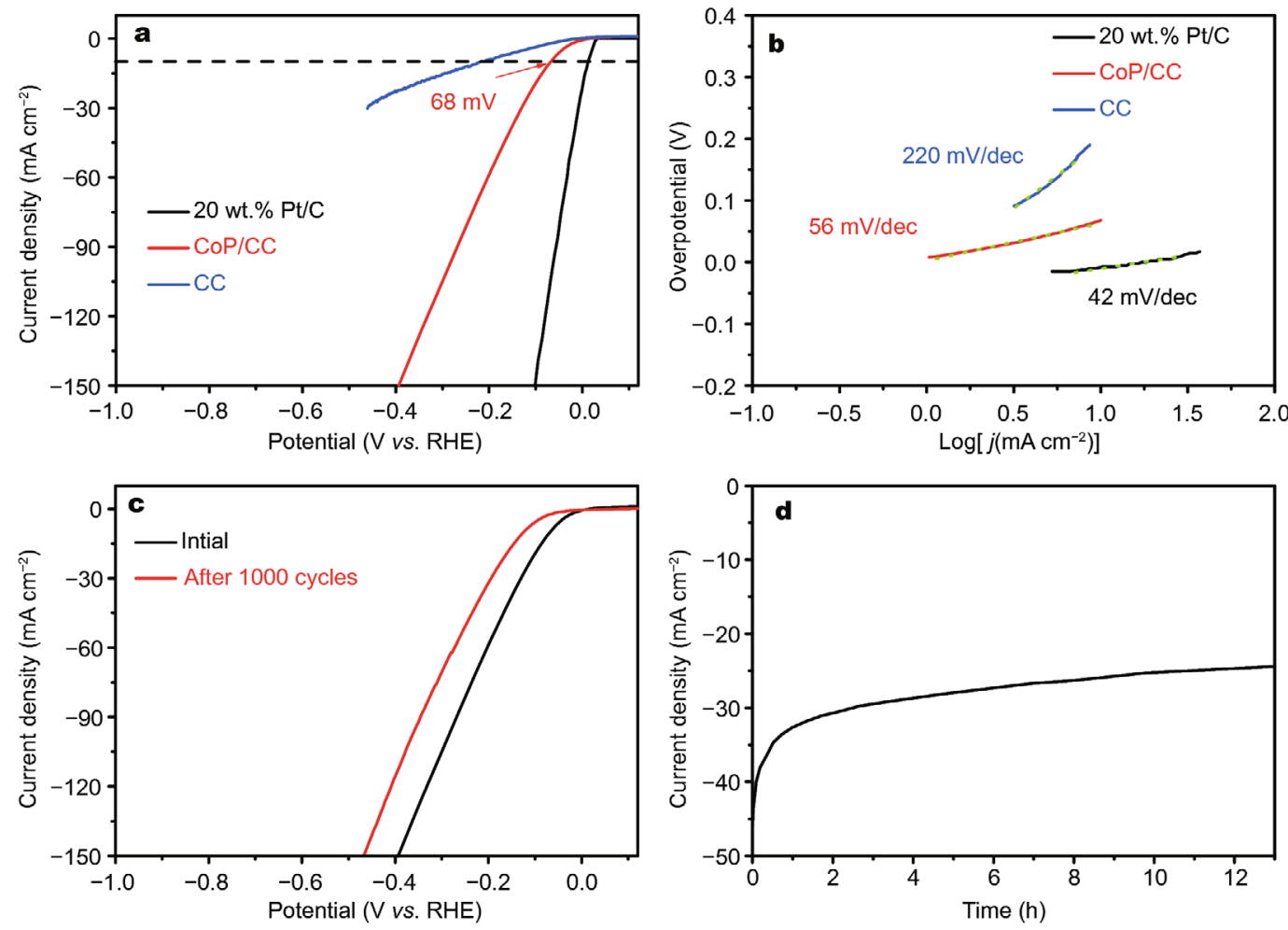

Figure 4 HER activity for CoP/CC, 20 wt.\% Pt/C and CC. (a) LSV curves of CoP/CC, 20 wt.\% Pt/C and CC in $0.5 \mathrm{~mol} \mathrm{~L}^{-1} \mathrm{H}_{2} \mathrm{SO}_{4}$ solution; (b) Tafel plots for CoP/CC, 20 wt.\% Pt/C and CC; (c) LSV of CoP/CC, initially and after 1000 cycles; (d) Time-dependent current density for CoP/CC under a static overpotential of $240 \mathrm{mV}$.

indicating that plenty of pores appear in the CoP nanowires. The large number of pores can promote the capacitance of the supercapacitor and provide electrocatalyst active sites for HER. The high-resolution TEM (HRTEM) image of the CoP nanowires is shown in Fig. 3f; a lattice fringe with an interplanar spacing of $2.8 \AA$, corresponding to the (011) plane of CoP nanowires, is observed. The CoP/CC can be used as electrocatalyst for HER and electrode material for supercapacitor.

The electrocatalytic activity of $\mathrm{CoP} / \mathrm{CC}$ for HER was investigated in $\mathrm{N}_{2}$-saturated $0.5 \mathrm{~mol} \mathrm{~L}^{-1} \mathrm{H}_{2} \mathrm{SO}_{4}$ electrolyte solution, using a standard three-electrode setup. For comparison, CC and $20 \mathrm{wt} . \% \mathrm{Pt} / \mathrm{C}$ were also examined under the same conditions. The LSV curves of CoP/CC, 20 wt.\% Pt/C and CC are presented in Fig. 4a, at a scan rate of $5 \mathrm{mV} \mathrm{s}^{-1}$. It can be clearly seen that the $20 \mathrm{wt} . \% \mathrm{Pt} /$ C presents excellent HER activity, with a low overpotential. The LSV curve of the CoP/CC demonstrates a low overpotential of $\sim 68 \mathrm{mV}$ at a current density of $10 \mathrm{~mA} \mathrm{~cm}^{-2}$, which compares favorably to most reported values for $\mathrm{CoP}$ and other non-noble metal HER catalysts in $0.5 \mathrm{~mol} \mathrm{~L}^{-1} \mathrm{H}_{2} \mathrm{SO}_{4}$ [14]. Finally, CC demonstrates poor HER activity, with an onset overpotential of about 219 $\mathrm{mV}$ at a current density of $10 \mathrm{~mA} \mathrm{~cm}{ }^{-2}$. The Tafel slopes of CoP/CC, 20 wt.\% Pt/C and CC could be obtained from the LSV curves; the Tafel plots are shown in Fig. 4b. The Tafel slope of the CC is calculated to be $220 \mathrm{mV} / \mathrm{dec}$, which is significantly higher than that of $\mathrm{CoP} / \mathrm{CC}$ and 20 wt.\% Pt/C, for which Tafel slopes of 56 and $42 \mathrm{mV} / \mathrm{dec}$, respectively, were calculated. The CoP/CC exhibit a low Tafel slope, which is close to that of $\mathrm{Pt} / \mathrm{C}$, confirming their high HER activity. Therefore, the HER activity of $\mathrm{CoP} / \mathrm{CC}$ mainly arises from the $\mathrm{CoP}$ and not the CC. For comparison, the LSV curve and Tafel plot of the electrocatalytic activity for HER of the precursor $\mathrm{Co}(\mathrm{OH}) \mathrm{F}$ are shown in Fig. S6, indicating that the $\mathrm{Co}(\mathrm{OH}) \mathrm{F}$ have a poor electrocatalytic performance for HER. In order to investigate the durability of $\mathrm{CoP} / \mathrm{CC}$, a CV scan between 0 and $-0.38 \mathrm{~V} v s$. RHE at a scan rate of $100 \mathrm{mV} \mathrm{s}^{-1}$ in 0.5 $\mathrm{mol} \mathrm{L}^{-1} \mathrm{H}_{2} \mathrm{SO}_{4}$, and the time-dependent current density were measured under a static overpotential of $240 \mathrm{mV}$. LSV curves of the initial and the $1000^{\text {th }}$ cycle are shown in Fig. $4 c$, indicating that the LSV curve of the $1000^{\text {th }}$ cycle 

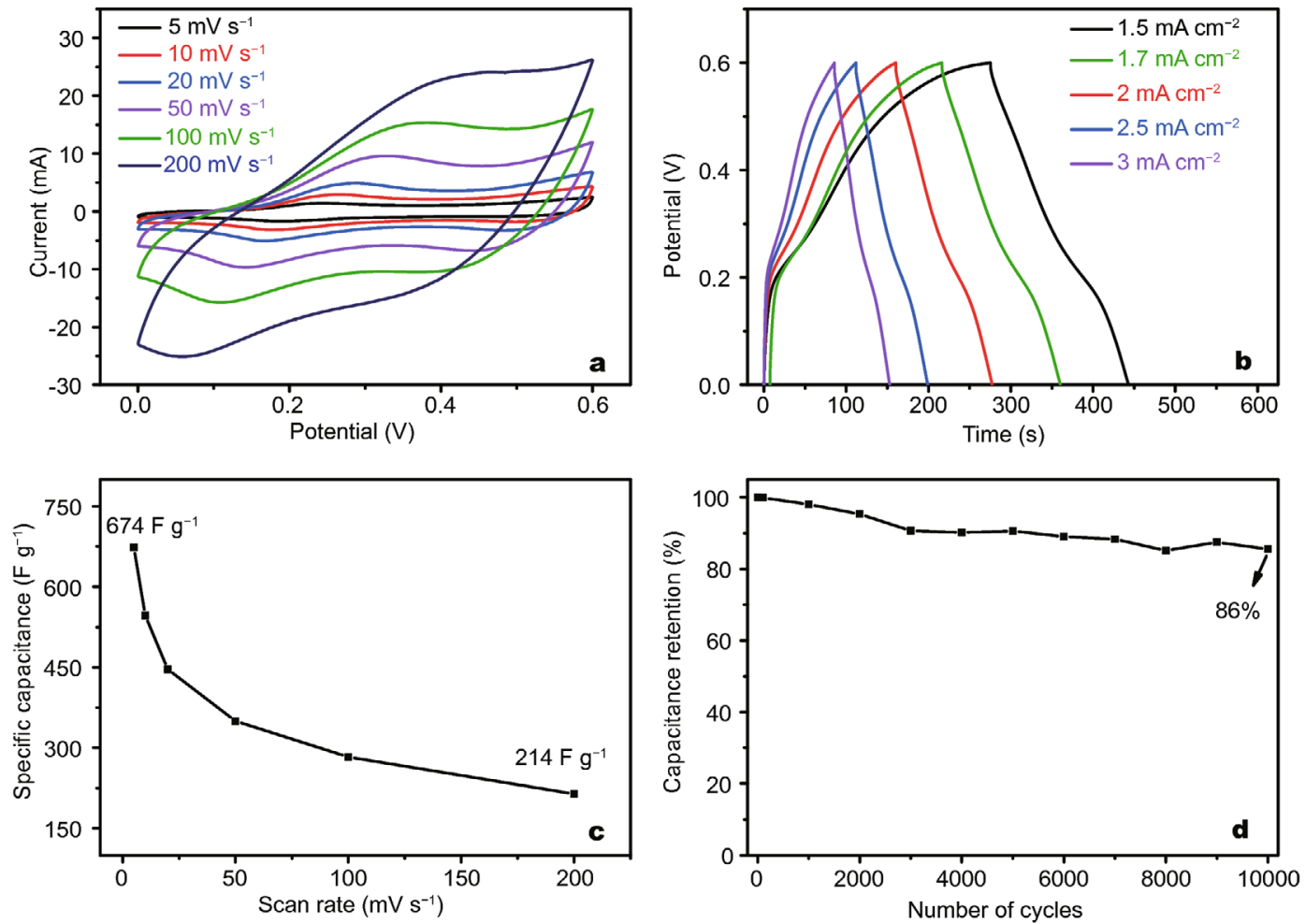

Figure 5 Supercapacitor performance of CoP/CC. (a) CV curves of CoP/CC at different scan rates; (b) Galvanostatic charging/discharging of CoP/ $\mathrm{CC}$ at different current densities; (c) Specific capacitance of CoP/CC at different scan rates; (d) Cycling performance of the CoP/CC electrode at a current density of $10 \mathrm{~mA} \mathrm{~cm}^{-2}$.

exhibits some differences compared to that of the initial curve. The overpotential of CoP/CC slowly drops from 68 to $124 \mathrm{mV}$ after 1000 cycles, indicating that the electrocatalytic activity of CoP/CC for HER is slightly lowered. This may be due to the fact that $\mathrm{H}_{2}$ bubbles retained on the surface of the CoP/CC also block the active sites $[23,31]$. Furthermore, the time-dependent current density of CoP/CC under a static overpotential of $240 \mathrm{mV}$ for $13 \mathrm{~h}$ is shown in Fig. $4 \mathrm{~d}$. After $13 \mathrm{~h}$, the current density of $\mathrm{CoP} / \mathrm{CC}$ has only reduced a little, indicating the good stability of CoP/CC as electrocatalyst for HER. The HER performances of transition metal phosphides are summarized in Table S1. Compared with recent reports, CoP/ CC exhibit excellent HER performance, with a low overpotential and small Tafel slope. Therefore, CoP/CC have great potential to be used as electrocatalyst for HER.

Very interestingly, CoP/CC can also be used as electrode material for supercapacitor. To evaluate the supercapacitor performance of the as-prepared CoP/CC, the $\mathrm{CoP} / \mathrm{CC}$ were tested as a working electrode in $1 \mathrm{~mol} \mathrm{~L}^{-1}$ $\mathrm{KOH}$. CV scans of CoP/CC as electrode in $1 \mathrm{~mol} \mathrm{~L}^{-1}$ $\mathrm{KOH}$ electrolyte at different scan rates $\left(5-200 \mathrm{mV} \mathrm{s}^{-1}\right)$, with a potential window from 0 to $0.6 \mathrm{~V}$, are shown in
Fig. 5a. A pair of redox peaks is observed during the charge and the discharge scans. As the scan rate is increased, the current density increases, and the anodic and cathodic peaks shift towards lower and higher potential, respectively. The shape of the redox peaks indicates that Faradaic reactions of CoP have occurred in the potential window. It is well known that the specific capacitance increases with current density, as the specific capacitance is proportional to the internal area of the CV curve. There is no obvious change in the $\mathrm{CV}$ curves as the scan rate is increased, which implies that the CoP/CC electrode has fast charge-discharge performances. The reversible capacity can be mainly attributed to the reversible reaction of $\mathrm{Co}^{2+} / \mathrm{Co}^{3+}$ and $\mathrm{Co}^{3+} / \mathrm{Co}^{4+}$. These results indicate that $\mathrm{CoP} / \mathrm{CC}$ as electrode material exhibit high capacitance. The galvanostatic charge/discharge curves of CoP/CC as electrode were tested at different current densities, in the potential range of 0 to $0.6 \mathrm{~V}$ (Fig. 5b). It is obvious that the galvanostatic charge/discharge curves are nonlinear and asymmetrical. Therefore, CoP/CC as electrode exhibit pseudocapacitive behavior [32]. The specific capacitance $\left(\mathrm{F} \mathrm{g}^{-1}\right)$ can be calculated from the $\mathrm{CV}$ curve. The specific capacitance of $\mathrm{CoP} / \mathrm{CC}$ as a function of the scan 
rate is presented in Fig. 5c. The specific capacitance of $\mathrm{CoP} / \mathrm{CC}$ as electrode is $674 \mathrm{~F} \mathrm{~g}^{-1}$ at a scan rate of 5 $\mathrm{mV} \mathrm{s}^{-1}$, which is higher than that of flower-like $\mathrm{MoS}_{2} / \mathrm{CC}$ nanosheets $\left(233 \mathrm{~F} \mathrm{~g}^{-1}\right), \mathrm{CoS}_{2} / \mathrm{CC}$ nanospheres $\left(266 \mathrm{~F} \mathrm{~g}^{-1}\right)$, $\mathrm{MoS}_{2} / \mathrm{CoS}_{2} / \mathrm{CC}$ nanocomposites (406 $\mathrm{F} \mathrm{g}^{-1}$ ) [10] and $\mathrm{MoS}_{2}\left(112 \mathrm{~F} \mathrm{~g}^{-1}\right)$ [33]. When the scan rate is increased to $200 \mathrm{mV} \mathrm{s}^{-1}$, the specific capacitance of CoP/CC as electrode is maintained at about $214 \mathrm{~F} \mathrm{~g}^{-1}$, confirming the good rate capability of CoP/CC. The cycling stability of the specific capacitance is one of most important characteristics of supercapacitor electrode materials. The long-term cycling stability of $\mathrm{CoP} / \mathrm{CC}$ as electrode was determined from galvanostatic charging/discharging curves at a current density of $10 \mathrm{~mA} \mathrm{~cm}^{-2}$ in $1 \mathrm{~mol} \mathrm{~L}^{-1}$ $\mathrm{KOH}$ electrolyte for 10,000 cycles (as shown in Fig. 5d). The capacitance retention is $86 \%$, even after 10,000 cycles, indicating the high cycling stability of CoP/CC. For comparison, the $\mathrm{CV}$ curves and specific capacitance as a function of scan rate of $\mathrm{CC}$ and $\mathrm{Co}(\mathrm{OH}) \mathrm{F} / \mathrm{CC}$ are shown in Figs S7 and S8, respectively. These results indicate that $\mathrm{CoP} / \mathrm{CC}$ perform better as electrode material than CC and $\mathrm{Co}(\mathrm{OH}) \mathrm{F}$. The high electrical conductivity of $\mathrm{CoP}$ can provide transportation paths for the electrolyte during Faradic reactions. The EIS data for CoP/CC, CC, and $\mathrm{Co}(\mathrm{OH}) \mathrm{F} / \mathrm{CC}$ are shown in Fig. S9. The gradual decline in capacitance is mainly attributed to $\mathrm{CoP}$ falling off the CC. The CoP/CC as electrode have an excellent electrochemical performance, as shown in Fig. 5, which demonstrates that CoP/CC are a practical electrode material suitable for potential application in supercapacitor.

\section{CONCLUSIONS}

In summary, we have successfully synthesized CoP nanowires supported on carbon cloth as a bifunctional electrode for HER and supercapacitor. The CoP/CC are quickly fabricated by a two-step procedure, which consists of a hydrothermal method followed by low-temperature phosphorization. These results indicate that CoP/CC as electrode exhibit excellent electrochemical performance for HER and supercapacitor. The overpotential of $\mathrm{CoP} / \mathrm{CC}$ is $68 \mathrm{mV}$ at a current density of 10 $\mathrm{mA} \mathrm{cm}{ }^{-2}$, which compares favorably with that of $20 \mathrm{wt} . \%$ $\mathrm{Pt} / \mathrm{C}$. The current density of CoP/CC only slowly decreases over $13 \mathrm{~h}$, indicating the good stability of CoP/CC as electrocatalyst for HER. When CoP/CC are used as electrode material for supercapacitor, the specific capacitance of CoP/CC as electrode is $674 \mathrm{~F} \mathrm{~g}^{-1}$ at a scan rate of $5 \mathrm{mV} \mathrm{s}^{-1}$. The high cycling stability of CoP/CC has been demonstrated; $86 \%$ of the initial capacitance is maintained after 10,000 cycles under a current density of
$10 \mathrm{~mA} \mathrm{~cm}^{-2}$ in $1 \mathrm{~mol} \mathrm{~L}^{-1} \mathrm{KOH}$ electrolyte. These results indicate the good electrochemical performance of $\mathrm{CoP} /$ $\mathrm{CC}$ as electrode for supercapacitor. This work puts forward a new viewpoint; non-noble metal material might be a promising candidate as electrode for HER and supercapacitor.

Received 30 June 2017; accepted 5 September 2017;

published online 26 October 2017

1 Chow J, Kopp RJ, Portney PR. Energy resources and global development. Science, 2003, 302: 1528-1531

2 Dresselhaus MS, Thomas IL. Alternative energy technologies. Nature, 2001, 414: 332-337

3 Tang C, Zhang R, Lu W, et al. Fe-doped CoP nanoarray: a monolithic multifunctional catalyst for highly efficient hydrogen generation. Adv Mater, 2017, 29: 1602441

4 Yu J, Li Q, Chen N, et al. Carbon-coated nickel phosphide nanosheets as efficient dual-electrocatalyst for overall water splitting. ACS Appl Mater Interfaces, 2016, 8: 27850-27858

5 Guo Y, Ren T. When it is unfamiliar to me: Local acceptance of planned nuclear power plants in China in the post-fukushima era. Energ Policy, 2017, 100: 113-125

6 Lovins AB. Nuclear power: deployment speed. Science, 2016, 354: $1112-1113$

7 Sanz J, Sobrados I, Soria J, et al. Anatase nanoparticles boundaries resulting from titanium tetrachloride hydrolysis. Catal Today, 2017, 281: 198-204

8 Giallanza A, Porretto M, Cannizzaro L, et al. Analysis of the maximization of wind turbine energy yield using a continuously variable transmission system. Renew Energ, 2017, 102: 481-486

9 Mestres M, Griñó M, Sierra JP, et al. Analysis of the optimal deployment location for tidal energy converters in the mesotidal Ria de Vigo (NW Spain). Energy, 2016, 115: 1179-1187

10 Su C, Xiang J, Wen F, et al. Microwave synthesized three-dimensional hierarchical nanostructure $\mathrm{CoS}_{2} / \mathrm{MoS}_{2}$ growth on carbon fiber cloth: a bifunctional electrode for hydrogen evolution reaction and supercapacitor. Electrochim Acta, 2016, 212: 941-949

11 Zheng Y, Jiao Y, Zhu Y, et al. Hydrogen evolution by a metal-free electrocatalyst. Nat Commun, 2014, 5: 3783

12 Zhuang $\mathrm{M}$, Ou X, Dou Y, et al. Polymer-embedded fabrication of $\mathrm{Co}_{2} \mathrm{P}$ nanoparticles encapsulated in N,P-doped graphene for hydrogen generation. Nano Lett, 2016, 16: 4691-4698

13 Wang X, Li W, Xiong D, et al. Bifunctional nickel phosphide nanocatalysts supported on carbon fiber paper for highly efficient and stable overall water splitting. Adv Funct Mater, 2016, 26: 4067-4077

14 Yang $\mathrm{H}$, Zhang $\mathrm{Y}$, Hu F, et al. Urchin-like CoP nanocrystals as hydrogen evolution reaction and oxygen reduction reaction dualelectrocatalyst with superior stability. Nano Lett, 2015, 15: 76167620

15 Stephens IEL, Chorkendorff I. Minimizing the use of platinum in hydrogen-evolving electrodes. Angew Chem Int Ed, 2011, 50: 1476-1477

16 Chua XJ, Luxa J, Eng AYS, et al. Negative electrocatalytic effects of p-doping niobium and tantalum on $\mathrm{MoS}_{2}$ and $\mathrm{WS}_{2}$ for the hydrogen evolution reaction and oxygen reduction reaction. ACS Catal, 2016, 6: 5724-5734

17 Feng JX, Ding LX, Ye SH, et al. Co(OH)@ @PANI hybrid nanosheets 
with 3D networks as high-performance electrocatalysts for hydrogen evolution reaction. Adv Mater, 2015, 27: 7051-7057

18 Yang H, Wang C, Hu F, et al. Atomic-scale Pt clusters decorated on porous $\alpha-\mathrm{Ni}(\mathrm{OH})_{2}$ nanowires as highly effcient electrocatalyst for hydrogen evolution reaction. Sci China Mater, 2017, https:// doi.org/10.1007/s40843-017-9035-8

19 Hou CC, Cao S, Fu WF, et al. Ultrafine CoP nanoparticles supported on carbon nanotubes as highly active electrocatalyst for both oxygen and hydrogen evolution in basic media. ACS Appl Mater Interfaces, 2017, 7: 28412-28419

$20 \mathrm{Pu} \mathrm{Z}$, Liu Q, Tang C, et al. $\mathrm{Ni}_{2} \mathrm{P}$ nanoparticle films supported on a Ti plate as an efficient hydrogen evolution cathode. Nanoscale, 2014, 6: 11031-11034

21 Tian J, Liu Q, Liang Y, et al. FeP nanoparticles film grown on carbon cloth: an ultrahighly active 3D hydrogen evolution cathode in both acidic and neutral solutions. ACS Appl Mater Interfaces, 2014, 6: 20579-20584

22 Cai Z, Song X, Wang Y, et al. Electrodeposition-assisted synthesis of $\mathrm{Ni}_{2} \mathrm{P}$ nanosheets on $3 \mathrm{D}$ graphene/Ni foam electrode and its performance for electrocatalytic hydrogen production. ChemElectroChem, 2015, 2: 1665-1671

23 Oh SK, Kim HW, Kwon YK, et al. Porous Co-P foam as an efficient bifunctional electrocatalyst for hydrogen and oxygen evolution reactions. J Mater Chem A, 2016, 4: 18272-18277

24 Wang $\mathrm{AL}$, $\mathrm{Lin} \mathrm{J}, \mathrm{Xu} \mathrm{H}$, et al. $\mathrm{Ni}_{2} \mathrm{P}-\mathrm{CoP}$ hybrid nanosheet arrays supported on carbon cloth as an efficient flexible cathode for hydrogen evolution. J Mater Chem A, 2016, 4: 16992-16999

25 Padmanathan N, Selladurai S, Xiang J. Sonochemically precipitated spinel $\mathrm{Co}_{3} \mathrm{O}_{4}$ and $\mathrm{NiCo}_{2} \mathrm{O}_{4}$ nanostructures as an electrode materials for supercapacitor. AIP Conference Proceedings, 2013, 512: 12161217

$26 \mathrm{Xu} \mathrm{D}, \mathrm{Mu}$ C, Xiang J, et al. Carbon-encapsulated $\mathrm{Co}_{3} \mathrm{O}_{4} @ \mathrm{CoO} @ \mathrm{Co}$ nanocomposites for multifunctional applications in enhanced long-life lithium storage, supercapacitor and oxygen evolution reaction. Electrochim Acta, 2016, 220: 322-330

27 Liu J, Cao H, Jiang B, et al. Newborn 2D materials for flexible energy conversion and storage. Sci China Mater, 2016, 59: 459-474

28 Tian J, Liu Q, Asiri AM, et al. Self-supported nanoporous cobalt phosphide nanowire arrays: an efficient 3D hydrogen-evolving cathode over the wide range of $\mathrm{pH} 0-14$. J Am Chem Soc, 2014, 136: $7587-7590$

29 Yang Y, Xu X, Wang X. Synthesis of Mo-based nanostructures from organic-inorganic hybrid with enhanced electrochemical for water splitting. Sci China Mater, 2015, 58: 775-784

30 Peng S, Li L, Han X, et al. Cobalt sulfide nanosheet/graphene/ carbon nanotube nanocomposites as flexible electrodes for hydrogen evolution. Angew Chem, 2014, 126: 12802-12807

31 Xie J, Zhang H, Li S, et al. Defect-rich $\mathrm{MoS}_{2}$ ultrathin nanosheets with additional active edge sites for enhanced electrocatalytic hydrogen evolution. Adv Mater, 2013, 25: 5807-5813

32 Kong D, Ren W, Cheng C, et al. Three-dimensional $\mathrm{NiCo}_{2} \mathrm{O}_{4} @$ polypyrrole coaxial nanowire arrays on carbon textiles for highperformance flexible asymmetric solid-state supercapacitor. ACS Appl Mater Interfaces, 2015, 7: 21334-21346

33 Ilanchezhiyan P, Mohan Kumar G, Kang TW. Electrochemical studies of spherically clustered $\mathrm{MoS}_{2}$ nanostructures for electrode applications. J Alloys Compd, 2015, 634: 104-108

Acknowledgements This work was supported by the National Natural Science Foundation of China (51732010, 51571172, 11404280, 51672240 and 51571171), the Natural Science Foundation for Distinguished Young Scholars of Hebei Province (E2017203095), the Natural Science Foundation of Hebei Province (E2016203484 and A2015203337), the Research Program of the College Science \& Technology of Hebei Province (ZD2017083 and QN2014047), and the Graduate Innovation Fund (CXZZSS2017055 and 2017XJSS044).

Author contributions Song J performed the experiments with help from Zhang $\mathrm{C}$ and Wang $\mathrm{C} . \mathrm{Mu} \mathrm{C}$ wrote the manuscript with support from Xiang J, Wang B, Wen F, and Liu Z. Xiang J, Mu C, Wang B, Wen $\mathrm{F}$, and Liu $\mathrm{Z}$ provided technical guidance and helped analyze the data. All author contributed to the general discussion.

Conflict of interest The authors declare that they have no conflict of interest.

Supplementary information online version of the paper.
Supporting data are available in the 


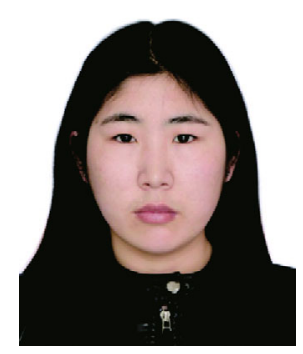

Jiefang Song is a master candidate under the supervision of Dr. Congpu Mu at Yanshan University. She received her bachelor's degree in 2015. Her research focuses on the synthesis and design of function materials.

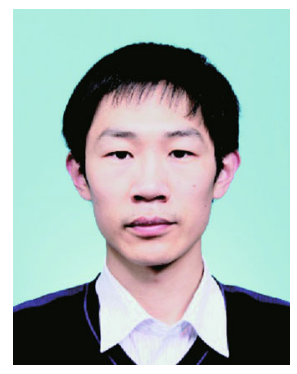

Congpu Mu was born in 1984 and joined Yanshan University in 2013. He completed his PhD in physics at Lanzhou University in 2013. His research is related to magnetic nanomaterials, from magnetic metals to magnetic oxide with their applications in microwave absorption and energy storage.

\section{碳布上快速合成 CoP纳米线阵列用于析氢和超级电容器}

宋杰芳 ${ }^{1,2}$, 向建勇 ${ }^{1}$, 牟从普 ${ }^{*}$, 王博羽 ${ }^{2}$, 温福昇 ${ }^{1,2^{*}}$, 苏灿 $^{2}$, 王聪 ${ }^{2}$, 柳忠元 $2^{2^{*}}$

摘要 本文利用微波辅助水热法在碳布上快速合成了 $\mathrm{CoP}$ 纳米线阵列 (CoP/CC)双功能电极材料, 可用于析氢反应(HER)和超级电容器的 电极. CoP/CC 用于HER时展现了优越的电化学性能, 其塔菲尔斜率为 $56 \mathrm{mV} / \mathrm{dec}$. 当电流密度为 $10 \mathrm{~mA} \mathrm{~cm}^{-2}$ 时, 其电位低至 $68 \mathrm{mV}$. 值得 注意的是, $\mathrm{CoP} / \mathrm{CC}$ 也可用于超级电容器的电极. 在扫描速率为 $5 \mathrm{mV} \mathrm{s}^{-1}$ 时, 其比电容高达 $674 \mathrm{~F} \mathrm{~g}^{-1}$. 同时具有高的循环稳定性, 在循环 10000 次后, 比电容仍然可以维持在初始值的 $86 \%$. 本文结果表明, CoP/CC可以作为HER和超级电容器电极的有力候选者. 